This document is confidential and is proprietary to the American Chemical Society and its authors. Do not copy or disclose without written permission. If you have received this item in error, notify the sender and delete all copies.

\title{
Intrafibrillar Mineralization of Self-Assembled Elastin-Like Recombinamer Fibrils
}

\begin{tabular}{|r|l|}
\hline Journal: & ACS Applied Materials \& Interfaces \\
\hline Manuscript ID & am-2016-15285q.R1 \\
\hline Manuscript Type: & Article \\
\hline Date Submitted by the Author: & $\mathrm{n} / \mathrm{a}$ \\
\hline Complete List of Authors: & $\begin{array}{l}\text { Li, Yuping; University of Minnesota, Restorative Sciences } \\
\text { Rodriguez-Cabello, José; Universidad de Valladolid, GIR Bioforge, Dept } \\
\text { Física de la Materia Condensada } \\
\text { Aparicio, Conrado; University of Minnesota, Minnesota Dental Research } \\
\text { Center for Biomaterials and Biomechanics }\end{array}$ \\
\hline
\end{tabular}

\section{SCHOLARONE \\ Manuscripts}


Intrafibrillar Mineralization of Self-Assembled Elastin-Like Recombinamer Fibrils

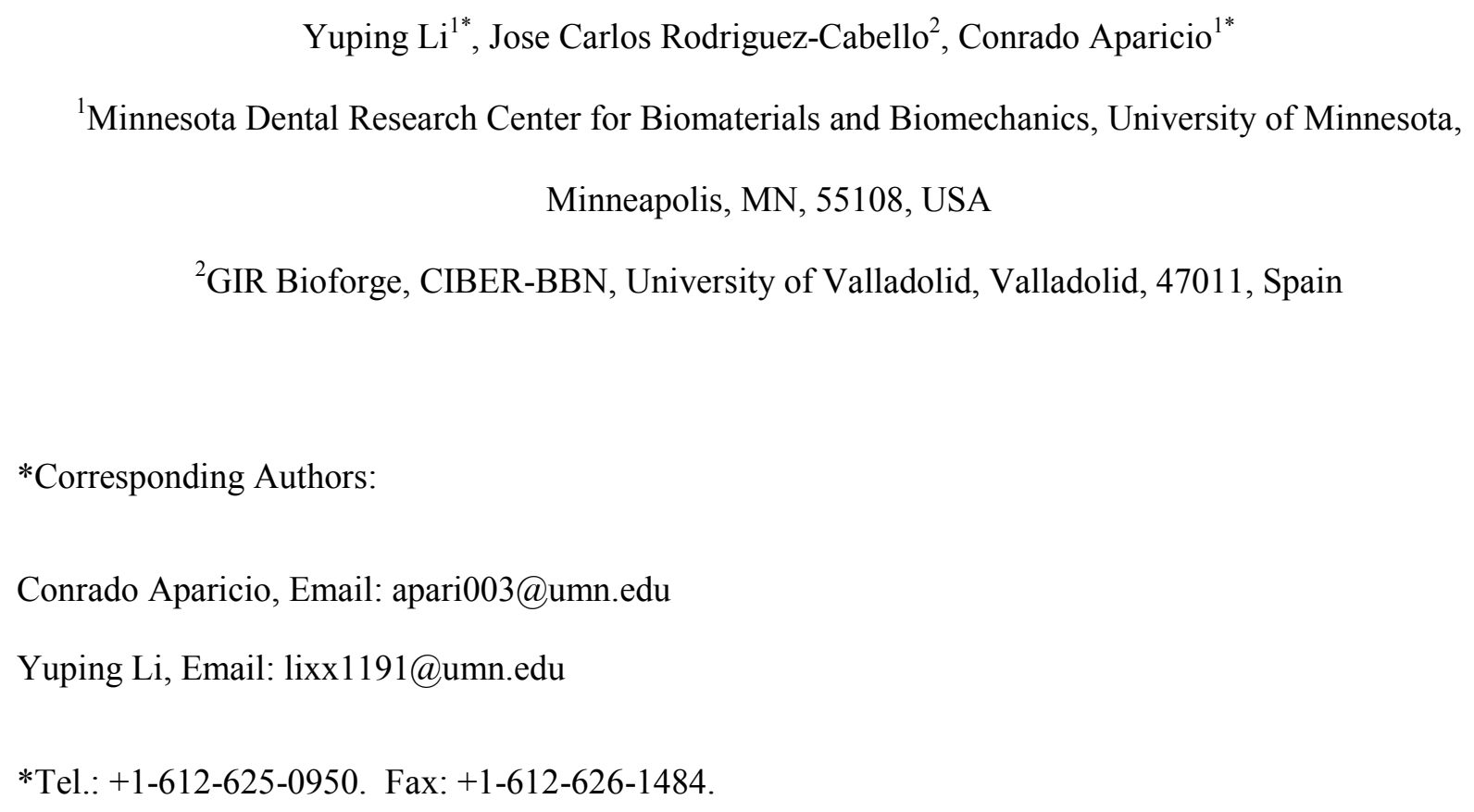


Abstract:

Biomineralization of bone, a controlled process where hydroxyapatite nanocrystals preferentially deposit in collagen fibrils, is achieved by the interplay of the collagen matrix and non-collagenous proteins. Mimicking intrafibrillar mineralization in synthetic systems is highly attractive for the development of advanced hybrid materials with elaborated morphologies and outstanding mechanical properties, as well as understanding the mechanisms of biomineralization. Inspired by nature, intrafibrillar mineralization of collagen fibrils has been successfully replicated in vitro via biomimetic systems where acidic polymeric additives are used as analog of non-collagenous proteins in mediating mineralization. The development of synthetic templates that mimic the structure and functions of collagenous matrix in mineralization has yet to be explored. In this study, we demonstrated that self-assembled fibrils of elastin-like recombinamers (ELRs) can induce intrafibrillar mineralization. The ELRs displayed a disordered structure at low temperature but self-assembled into nanofibrils above its inverse transition temperature. In the presence of the self-assembled ELR fibrils, polyaspartate-stabilized amorphous calcium phosphates ]preferentially infiltrated into the fibrils and then crystalized into hydroxyapatite crystals with their [001] axes aligned parallel to the long axis of the ELR fibril. As the recombinant technology enables designing and producing well-defined ELRs, their molecular and structural properties can be fine-tuned. By examining the ultrastructure of the self-assembled ELRs fibrils as well as their mineralization, we concluded that the spatial confinement formed by a continuum $\beta$-spiral structure in an unperturbed fibrillar structure rather than electrostatic interactions or bioactive sequences in the recombinamer composition played the crucial role in inducing intrafibrillar mineralization.

Keywords: Elastin-like recombinamer, biomimetic mineralization, calcification, collagen, amorphous calcium phosphate precursor, bone 


\section{Introduction}

Biomineralization of extra-cellular matrices (ECMs), a process for the formation of hard tissues, involves a controlled deposition of calcium phosphate minerals into an organic matrix, through which the mineralized tissues endow elaborate morphologies and outstanding mechanical properties. ${ }^{1-2}$ One of the most representative examples in biologically controlled mineralization is the intrafibrillarly mineralized type I collagen fibril, the basic building block of bone and dentin, in which collagen fibrils act as an organic template in directing the infiltration and orientation of hydroxyapatite (HAp) nanocrystals.-5 Investigations on intrafibrillar mineralization of collagen fibrils using in vivo and in vitro models have indicated that this process requires 1) collagen molecules self-assemble into a highly ordered structure that provides highly charged zones and nanoscale compartments for mineral infiltration, deposition and orientation; and 2) HAp crystals form via a polymer-induced liquid-like amorphous calcium phosphate (ACP) phase. ${ }^{4,6-8}$ Type I collagen is the most abundant ECM protein in vertebrates which consists of three left-handed polyproline peptide chains intertwined in a right-handed manner. ${ }^{9}$ The triple-helical collagen molecules are packed in a quasi-hexagonal mode to form 4-nm microfibrils which further bundle into fibrils. ${ }^{10-12}$ Collagen molecules align in a parallel and staggered array, showing a $67-\mathrm{nm}$ D-periodic banding pattern where the gap zone is $35 \mathrm{~nm}$ and the overlap zone is $32 \mathrm{~nm} .^{13}$ Many studies have indicated that acidic non-collagenous proteins (NCPs)—osteopontin, phosphorylated dentin phosphophoryn (DPP), fetuin and the C-terminal fragment of the dentin matrix protein 1 (C-DMP1)present in the ECMs, play an important role in mineralization by nucleating HAp crystallization, inhibiting crystal formation, or modulating crystal growth. ${ }^{14}$ Inspired by the role of NCPs in bone and dentin formation, intrafibrillar mineralization of collagen fibrils has been successfully reproduced in vitro using the polymer-induced liquid-precursor (PILP) process where polyaspartic acid (PASP) was used as a substitute of NCPs. ${ }^{45-16}$ Further studies on the in vitro mineralization model demonstrated that the PASP stabilizes ACP phase in the form of highly negatively charged nanoclusters which were preferentially attracted into the highly positively charged a-band of collagen fibrils located at their gap zones. ${ }^{17-18}$ 
Consequently, the liquid-like ACP nanoclusters solidify into HAp in the gap zone and then grow along the fibril that their c-axes are oriented nearly parallel along the long axis of the fibrils. ${ }^{4}$ While a number of attempts have been succeeded in duplicating the nanostructure of bone using negatively charged polymers as an analogue of the NCPs, the development of alternative organic matrices which can mimic structural features and functions of collagen in directing mineralization is still on the early stage. Besides, the role of collagen matrix in mineralization, in terms of controlling mineral infiltration and crystallization, as well as guiding the crystallographic orientation of the hydroxyapatite nanocrystals, has not been well understood yet.

The ability to achieve intrafibrillar mineralization in synthetic systems is also highly attractive as it helps to identify key strategies for controlled deposition of calcium phosphate minerals and provides guidance for the development of next generation hard tissue-related materials. A number of experiments have been carried out in an attempt to mimic mineralization of collagen fibrils using a polymeric matrix as an analogue of collagen and traditional mineralization processes, such as incubation in simulated body fluid. In most cases, only surface precipitation of calcium phosphate crystals can be obtained in synthetic matrices, e. g. polylactide (PLA), poly(lactic-co-glycolic acid) (PLGA) and polycaprolactone (PCL). ${ }^{19-21}$ In preliminary studies, epoxy resin and PMMA blocks were incubated in the PILP solution and no mineral was formed in these polymers either (data not shown). It is likely that the molecules in these polymeric matrices were so densely packed that the infiltration of minerals was highly inhibited. In contrast, the organic matrix with nanoporous structure seems to be the most promising structural template for mineralization as it provides nanoscale confinements for mineral infiltration within. Because collagen fibrils provide gap zones and interstitial spaces around collagen microfibrils for mineral deposition, it is reasonable that the intrafibrillar mineralization requires construction of the compartment or space to occur first in the fibril so it can regulate the mineral infiltration into the localized space. ${ }^{6,22}$

Tropoelastin and elastin-like polypeptides/recombinamers (ELRs) are thermally responsive molecules. Below a given temperature, i.e., the inverse transition temperature $\left(T_{t}\right)$, tropoelastin and its synthetic 
analogs - the ELRs, remain soluble and have their $\beta$-spirals in extended (unfolded) state. ${ }^{23}$ Upon Tt, the ELRs self-assemble into a hierarchical organization at multiple length scales ranging from folded $\beta$ spirals, 5-nm diameter filaments, to fibrils with a diameter less than a few hundred nanometers. ${ }^{24-26}$ The initial self-assembled structure, the so-called $\beta$-spiral, is of particular interest. Because of the helical recurrent formation of type II $\beta$-turns, one per each repeating (VPGXG) unit, it forms $\beta$-spiral immediately after the temperature goes above its $\mathrm{T}_{\mathrm{t} .}{ }^{27}$ This $\beta$-spiral is quite dynamic, much more than, e. g., an $\alpha$-helix, which is stabilized by inter-turn hydrogen bonds that do not exist in the $\beta$-spiral.

For the interest of this work and according to Urry et al., ${ }^{28}$ in the model $\beta$-spiral, each poly(VPGXG) macromolecule creates a 1.8-nm wide helix with a hydrophilic hollow core and a 1-nm periodic inter-turn along the helix. These $\beta$-spirals further associate into multi-stranded twisted filaments. In the presence of minerals, the interstitial spaces between these $\beta$-spirals might serve as the nanocompartment for mineral deposition and the organization of molecules along the ELR fibrils might direct crystal growth. The inclusion of a bioactive peptide domains in poly(VPGXG) influences the formation of the $\beta$-spiral and it may further affect the mineralization process. Note the bioactive sequence causes disturbance of the helical recurrent formation of type II $\beta$-turns. ${ }^{29}$ As shown in Table 1, REDV contains a short peptide (EEIQIGHIPREDVDYHLYP) with known bioactivity of promoting cell adhesion $^{28}$ but no specific activity on $\mathrm{CaP}$ mineralization apart from unspecific electrostatic interactions mediated by its charged side chains. Both HSS1 and HSS3 are the one and triblock versions of ELRs that contain the human salivary-derived peptide-DDDEEKFLRRIGRFG, known as $\mathrm{SN}_{\mathrm{A}} 15 . \mathrm{SN}_{\mathrm{A}} 15$ has specific binding activity with HAp. ${ }^{30}$ A previously study has shown that the ELRs containing the $\mathrm{SN}_{\mathrm{A}} 15$ peptide nucleated and promoted growth of HAp in solutions of calcium and phosphate at physiological conditions. ${ }^{31}$ Our previous study has also demonstrated that the CaP minerals preferentially deposited into the ELR hydrogels with controlled morphologies. ${ }^{32}$ As the hierarchical structure of ELRs (from amino acids, $\beta$-spiral, filament to fibril) is of particular analogy to that of collagen (from amino acids, triple-helix, microfibril to fibril), the differences in structure and potential interaction with calcium and 
phosphate of the ELRs tested here might help us understanding the mechanisms of biological mineralization as well as developing advanced materials for biomedical applications.

\section{Materials and Experimental Methods}

\subsection{Preparation of self-assembled ELR fibrils.}

The lyophilized ELR powders were dissolved in deionized water at $4{ }^{\circ} \mathrm{C}$ overnight to obtain solutions of 5 $\mathrm{mg} / \mathrm{ml}$ and $20 \mathrm{mg} / \mathrm{ml}$, respectively. They were then transferred into PTFE molds (dia. $4 \times 2 \mathrm{~mm}$ ) and incubated at $37^{\circ} \mathrm{C}$ for $20 \mathrm{~min}$ to induce self-assembly. After being taken out from an incubator, the molds were immediately immersed into liquid $\mathrm{N}_{2}$ and lyophilized overnight to obtain ELR sponges composed of self-assembled ELRs. The ELR sponges without self-assembly were prepared by directly lyophilizing the pure solution obtained at $4{ }^{\circ} \mathrm{C}$. Cross-linking of the ELR sponges was performed in a mixture of hexamethylene diisocyanate (HDMI) and acetone (1:9 v/v) overnight. After thoroughly rinse with acetone to remove HDMI, the cross-linked ELR sponges were rehydrated in a graded deionized water series $\left(50,70,90,95\right.$ and $100 \%$ of $\mathrm{H}_{2} \mathrm{O}$ in acetone) for 5 minutes each. The rehydrated ELR sponges were either directly placed into the PILP solution for mineralization or lyophilized overnight and stored at $-20^{\circ} \mathrm{C}$ before characterization.

\subsection{Mineralization of ELR Fibrils via the PILP Process.}

ELR fibrils, obtained from the aqueous solutions of $20 \mathrm{mg} / \mathrm{ml}$, were biomimetically mineralized with calcium phosphate via the polymer-induced liquid-precursor (PILP) process. Preparation of the PILP solution is detailed in the Supporting Information. The cross-linked ELR fibrils were incubated in $20 \mathrm{~mL}$ of the PILP solution at $37^{\circ} \mathrm{C}$ for mineralization and the PILP solution was refreshed every 3 days. After mineralization at the predetermined period, the ELRs were removed from the solution, thoroughly washed with deionized water and lyophilized for characterization.

\subsection{Characterization.}


Secondary structure of IK24 and the effects of temperature on its conformational change were investigated by circular dichroism spectropolarimetry (JASCO J-815 spectropolarimeter, JASCO Inc., Easton, MD) equipped with a temperature controller. The recombinant biopolymer solution (IK24, 0.3 $\mathrm{mg} / \mathrm{mL}$ in deionized water) was incubated in a $0.1-\mathrm{cm}$ path length quartz UV cuvette with a sealable cap. CD spectra were obtained by signal integration of 3 scans from 190 to $260 \mathrm{~nm}$ at a scan rate of $50 \mathrm{~nm} / \mathrm{min}$. The spectra were acquired from $15{ }^{\circ} \mathrm{C}$ to $45{ }^{\circ} \mathrm{C}$ with a $5{ }^{\circ} \mathrm{C}$ increment and vice versa. The solution was equilibrated at each temperature for $5 \mathrm{~min}$ before scanning. JASCO spectral analysis software was used to process CD data. FTIR analysis of the lyophilized ELR fibrils before and after mineralization was performed in a Thermo Scientific Nicollet iS50 FT-IR spectrometer, equipped with a built-in diamond attenuated total reflection (ATR) for single-spot ATR measurement. Each spectrum was the result of signal-averaging of 32 scans at a resolution of $4 \mathrm{~cm}^{-1}$, and the wavenumber ranged from 400 to $4000 \mathrm{~cm}^{-1}$. For the spectra used for deconvolution analysis, $128 \mathrm{scans}$ at a resolution of $2 \mathrm{~cm}^{-1}$ were applied. All spectra were transmittance spectra after background subtraction. Smoothing of spectra was carried out with a step of 11 data points, using the Savitzky-Golay Function. ${ }^{33}$ Second derivatives of the spectra were obtained using a step of 13 data points to identify discrete absorption bands that make up the complex amide profile. To measure the relative areas of the amide I components, peak fitting of the spectra was performed by using OriginLab Software (Microcal Inc.). The relative areas of the single bands were used to determine the fraction of the secondary structures.

Both semi-environmental and field-emission scanning electron microscopy (Hitachi TM-3000 and JEOL 6500, Japan) were used to analyze the morphology of self-assembled ELR fibrils before and after mineralization at an accelerating voltage of $15 \mathrm{kV}$ and $5 \mathrm{kV}$ respectively. For FE-SEM, the lyophilized samples were mounted on aluminum stubs with double-sided carbon tape and sputter coated with $5 \mathrm{~nm}$ Pt. A TEM (FEI Tecnai T12, Hillsboro, OR, USA) operated at $120 \mathrm{kV}$ was used to record TEM images of the specimens. Selected-area electron diffraction (SAED) and dark-field imaging were performed to identify the crystalline structure and the orientation of the crystals. The stained grids were rinsed with 
deionized water and air-dried. Ultrathin TEM sections $(\sim 80 \mathrm{~nm})$ of the mineralized IK24 fibrils were prepared by embedding them into epoxy resin, cut with a diamond knife on an ultramicrotome (Leica Reichert UltraCut S) and then collected on 300-mesh copper grids.

Crystal structure of the minerals was analyzed using a two-dimensional x-ray microdiffractometric system (Bruker AXS, Germany) operated at $45 \mathrm{kV}$ and $40 \mathrm{~mA}$. The incident angle was set at $15^{\circ}$ and detector position was fixed at $30^{\circ}$, which covered the angular range from 15 to $45^{\circ}$ in $2 \theta$. The data collection time was 1000 s and the results were analyzed using JADE8 software (Materials Data Inc, JADE, Livermore, CA, USA).

\section{Results and Discussion}

\subsection{Reversible thermal-responsive behavior and secondary structure of ELRs}

Five types of ELRs were used in this study. Their sequences and physicochemical properties are listed in Table 1. We first investigated the secondary structures and reversible thermal-responsive behavior of ELRs in aqueous solutions. Figure 1A and 1B show the thermally driven conformational transition of IK24 determined by CD. A strong negative band below $200 \mathrm{~nm}$ and a weak negative band near $220 \mathrm{~nm}$ are the typical spectral feature of the polyproline II left-handed helix (PPII) CD spectra. ${ }^{34}$ The intensity of the strong negative band at $198 \mathrm{~nm}$ was gradually reduced with the increase of temperatures (Figure 1A), indicating conformational transition toward a more folded state. This conformational transition is reversible since an opposite trend was observed by decreasing the temperature (Figure 1B).

Table 1. Physicochemical properties of the elastin-like recombinamers

\begin{tabular}{|c|c|c|c|}
\hline ELR & Sequence* & $M_{W}(k D a)$ & pI \\
\hline IK24 & $\left((\text { VPGIG })_{2}(\text { VPGKG)(VPGIG) })_{2}\right)_{24}-\mathrm{V}$ & 51.9 & 11.0 \\
\hline VK24 & $\left((\mathrm{VPGVG})_{2}(\mathrm{VPGKG})(\mathrm{VPGVG})_{2}\right)_{24}-\mathrm{V}$ & 49.9 & 11.9 \\
\hline REDV & $\begin{array}{l}{\left[(\mathrm{VPGIG})_{2}(\mathrm{VPGKG})(\mathrm{VPGIG})_{2} \text { EEIQIGHIPREDVDYHLYP }\right.} \\
\left.(\mathrm{VPGIG})_{2}(\mathrm{VPGKG})(\mathrm{VPGIG})_{2}(\mathrm{VGVAPG})_{3}\right]_{10}-\mathrm{V}\end{array}$ & 80.9 & 5.4 \\
\hline HSS1 & $\begin{array}{l}{\left[\left((\mathrm{VPGIG})_{2}(\mathrm{VPGKG})(\mathrm{VPGIG})_{2}\right)_{2} \text { DDDEEKFLRRIGRFG }\left((\mathrm{VPGIG})_{2}\right.\right.} \\
\left.\left.\text { VPGKG(VPGIG })_{2}\right)_{2}\right]_{3}-\mathrm{V}\end{array}$ & 31.9 & 9.9 \\
\hline HSS3 & $\begin{array}{l}{\left[\left((\mathrm{VPGIG})_{2}(\mathrm{VPGKG})(\mathrm{VPGIG})_{2}\right)_{2} \text { DDDEEKFLRRIGRFG }\left((\mathrm{VPGIG})_{2}\right.\right.} \\
\left.\left.(\mathrm{VPGKG})(\mathrm{VPGIG})_{2}\right)_{2}\right]_{3}(\mathrm{VPAVG})_{20}\left[\left((\mathrm{VPGIG})_{2}(\mathrm{VPGKG})(\mathrm{VPGIG})_{2}\right)\right. \\
\left.{ }_{2} \text { DDDEEKFLRRIGRFG }\left((\mathrm{VPGIG})_{2}(\mathrm{VPGKG})(\mathrm{VPGIG})_{2}\right)_{2}\right]_{3}-\mathrm{V}\end{array}$ & 71.5 & 10.1 \\
\hline
\end{tabular}


*bioactive sequences are marked by underlines
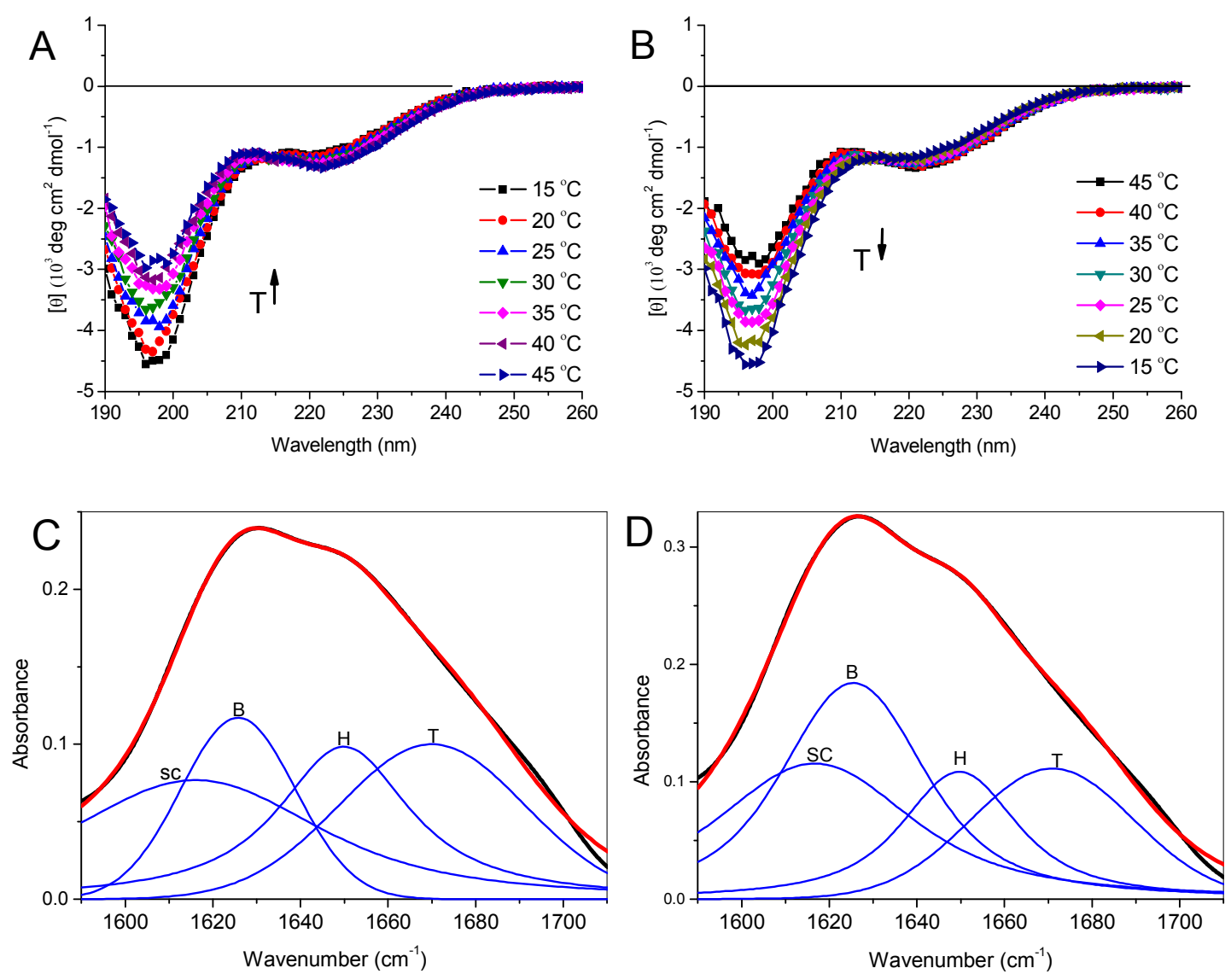

Figure 1. CD spectra of elastin-like recombinamers (IK24) in aqueous solution at temperatures of 15$45{ }^{\circ} \mathrm{C}$ through heating (A) and cooling (B) stepwise. Decomposed ATR-FTIR spectra of amide I region of IK24 obtained at $4{ }^{\circ} \mathrm{C}(\mathrm{C})$ and $37{ }^{\circ} \mathrm{C}$ (D). Experimental spectrum (black solid line), deconvoluted spectrum (blue solid line) and calculated spectrum (dashed red line). SC: beta-sheet aggregate, B: beta sheet, H: alpha-helix, T: beta-turn.

While CD studies investigated the secondary structure of IK24 in aqueous solution by highlighting the dominant component of PPII, ATR-FTIR studies allowed us to observe the conformational differences 
between ordered and amorphous forms of IK24 (Figure 1C and 1D). The amide I band of IK24 at both 4 and $37{ }^{\circ} \mathrm{C}$ showed a line shape of the $\beta$-spiral. The main absorption band at $1625 \mathrm{~cm}^{-1}$ is commonly assigned to $\beta$-sheet. ${ }^{35-37}$ The component around $1649 \mathrm{~cm}^{-1}$ is usually assigned to $\alpha$-helix structure. ${ }^{38}$ A low frequency component near $1616 \mathrm{~cm}^{-1}$ and a high frequency component near $1670 \mathrm{~cm}^{-1}$ are commonly assigned to $\beta$-sheet aggregates and $\beta$-turn, respectively. ${ }^{39}$ When the amide I band was deconvoluted using Fourier self-deconvolution (Table 2), a significant increase of intensity at $1625 \mathrm{~cm}^{-1}$ was detected, indicative of an increase of $\beta$-sheets, from $4{ }^{\circ} \mathrm{C}$ to $37{ }^{\circ} \mathrm{C}$. The intensity reduction of $1649 \mathrm{~cm}^{-1}$ and 1670 $\mathrm{cm}^{-1}$ is in support of a transition into a more folded state of $\beta$-spirals. ${ }^{38-39}$

Table 2. Peak positions and relative integrated intensities of deconvoluted amide I peak components of IK24 at $4{ }^{\circ} \mathrm{C}$ and $37{ }^{\circ} \mathrm{C}$.

\begin{tabular}{ccccc}
\hline & \multicolumn{2}{c}{$4{ }^{\circ} \mathrm{C}$} & \multicolumn{2}{c}{$37^{\circ} \mathrm{C}$} \\
\cline { 2 - 5 } Assignment & Position $\left(\mathrm{cm}^{-1}\right)$ & Area (\%) & Position $\left(\mathrm{cm}^{-1}\right)$ & Area (\%) \\
\hline $\boldsymbol{\beta}$-sheet aggregation & 1616 & 26.2 & 1616 & 26.1 \\
(SC) & 1625 & 20.9 & 1625 & 34.1 \\
$\boldsymbol{\beta}$-sheet (B) & 1649 & 24.6 & 1649 & 18.1 \\
$\boldsymbol{\alpha}$-helix (H) & 1670 & 28.2 & 1670 & 21.6 \\
$\boldsymbol{\beta}$-turns (T) & & &
\end{tabular}

\subsection{Fibrillar structure of the self-assembled ELR fibrils}

Tropoelastin and ELRs can self-aggregate into a packed fibrillar structure through thermally driven phase transition. $^{40-41}$ At the concentration of $20 \mathrm{mg} / \mathrm{ml}$, IK24 appeared as a film-like amorphous structure at $4{ }^{\circ} \mathrm{C}$ (Figure 2A), whereas they self-assembled into long fibrils with branches at $37{ }^{\circ} \mathrm{C}$ (Figure 2B). Subfibrillar structures, filaments, were observed on the TEM image, showing dark strands in the negatively stained IK24 fibril (Figure 2C). A fibrillar structure with a helical twisted rope-like substructure has been found from the heat aggregated poly(VPGVG). ${ }^{42-43}$ In our study, the appearance of PPII helix, $\beta$-sheet and $\beta$-turn found from the CD and FTIR indicated the existence of the $\beta$-spiral. ${ }^{44}$ Association of $\beta$-spirals by hydrophobic interactions resulted into the twisted filaments that were laterally 

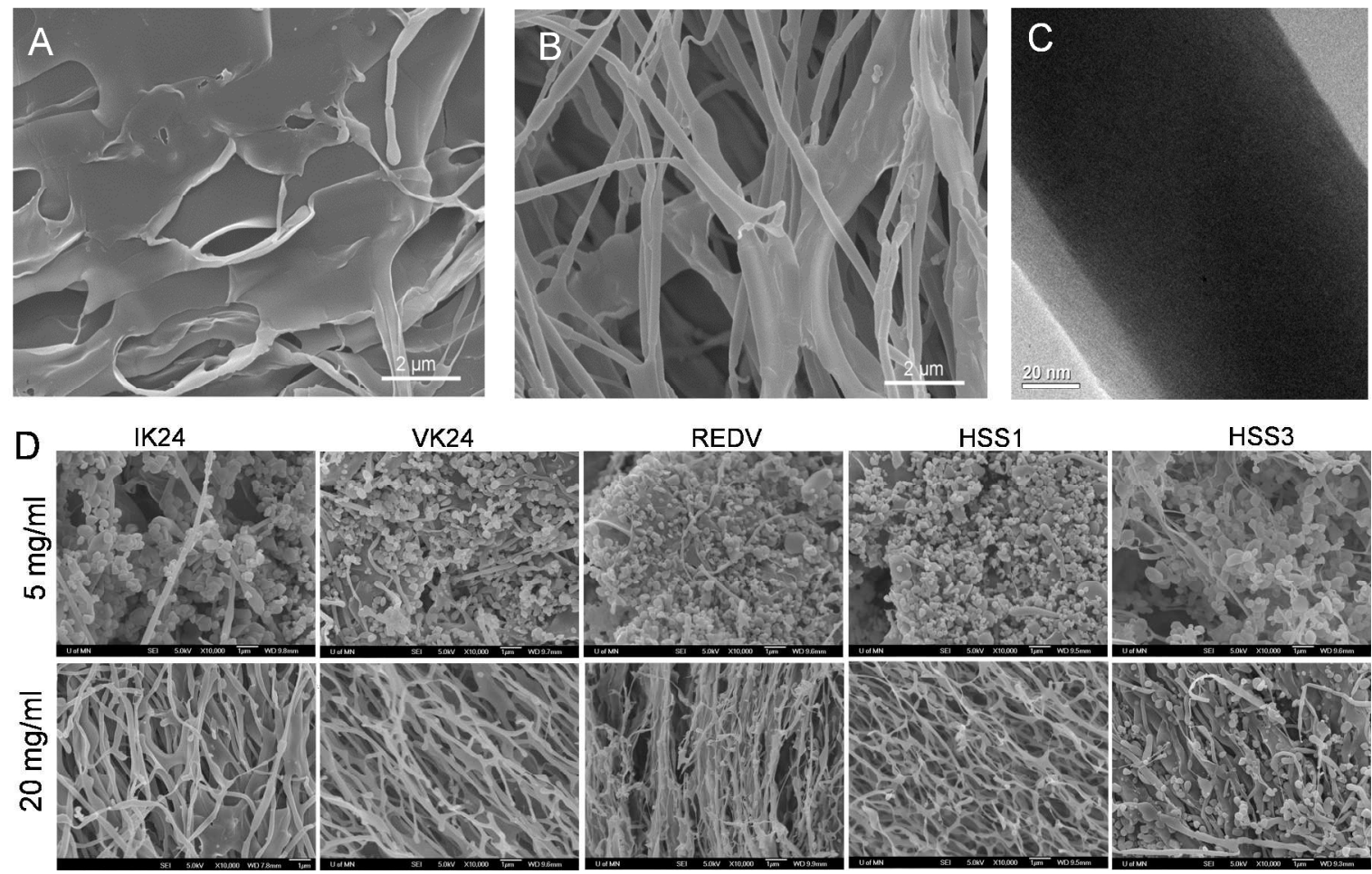

Figure 2. SEM images of the lyophilized IK24 (20 mg/ml) showing amorphous morphology at $4^{\circ} \mathrm{C}(\mathrm{A})$ and the fibrillar structure at $37^{\circ} \mathrm{C}$ (B). (C) TEM image of a cross-linked IK24 fibril stained with uranyl acetate showing dark strands along the fibril. (D) SEM images of ELRs which were self-assembled at $37^{\circ} \mathrm{C}$ in aqueous solutions at the concentration of $5 \mathrm{mg} / \mathrm{ml}$ (top row) and $20 \mathrm{mg} / \mathrm{ml}$ (bottom row).

All ELR studied here, IK24, VK24, REDV, HSS1 and HSS3, were self-assembled after 20 min of incubation at $37{ }^{\circ} \mathrm{C}$ (Figure 2D). At the low concentration, e. g. $5 \mathrm{mg} / \mathrm{ml}$, ELRs appeared primarily as globular clusters with diameters ranging from 100 to $400 \mathrm{~nm}$. They were self-organized into fibrillar networks at the concentration of $20 \mathrm{mg} / \mathrm{ml}$. It has been suggested that the fibrillar structure is determined by the $\operatorname{Gly}(\mathrm{G})$ and Pro(P) residues in pentapeptide sequences. ${ }^{27}$ Because VPGVG and VPGKG are the 
major sequences of the ELRs studied here, formation of fibrillar structure is in support of the role of these pentapetide sequences in superstructure organization. Globular spheres were also found in $20 \mathrm{mg} / \mathrm{ml}$ of HSS3. The observed granules might be on the process of structural rearrangement to form fibrils because they have also been found from poly(GVGAP), immature self-aggregated tropoelastin, and other recombinant elastin-like polypeptides. ${ }^{25}$

\subsection{Mineralization of the self-assembled ELR fibrils}

As the self-assembled ELR fibrils can be easily dissolved in aqueous solution, a chemical cross-linking reaction using isocyanate was performed to fix their fibrillar structure before biomimetic mineralization. After 7 days of mineralization, the fibrillar structure was still observed in all ELRs studied here (Figure 3A to 3E). IK24 and VK24 showed granular morphology from both the surface and the inner side of the fibrils indicating that the minerals have infiltrated within them. In contrast, the surface of REDV fibrils was also granulated but displayed a much smoother surface than that of IK24 and VK24. HSS1 and HSS3 showed needle-like crystals on their surface probably due to the overgrowth of the mineral. XRD confirmed that the minerals were HAp nanocrystals as the characteristic peaks of HAp, from the crystal planes of (002), (210), (211), (202), (130), (131) and (113), were observed (Figure 3F). ATR-FTIR spectroscopy analysis (Figure S2) on ELR fibrils before and after 7 days of mineralization revealed the appearance of strong peaks at 1022, 874, 601 and $560 \mathrm{~cm}^{-1}$ that were indicative of nonstoichiometric carbonated HAp crystallites. ${ }^{45-46}$

Although the mineralized fibrils were found in all five types of ELRs, it seems the fibrillar structure was not the stable state of the self-assembled REDV, HSS1 and HSS3. As shown in Figure S1, the mineralized REDV, HSS1 and HSS3 sponges were much denser than that of IK24 and VK24 suggesting the fibrils have coalesced before mineralization. This indicates that the incorporation of these short sequences hindered the lateral alignment for ELR fibril formation. The $\beta$-spiral shown in all ELRs is based on the basic sequence, poly(VPGXG), where X can be any L-amino acid. It forms regularly spaced $\beta$-turn around the P-G dipeptide which takes place along the polypeptide chain. These $\beta$-turns are caused 
by the peculiar conformational constrains of proline along with the non-bulking side chain of the glycine. Combination of chain-direction shifting in each $\beta$-turn and the regularity of these $\beta$-turns along the polypeptide backbone leads to the emergence of a regular and peculiar helical secondary structure named $\beta$-spiral. ${ }^{29}$ The presence of the charged bioactive domains, e.g. EEIQIGHIPREDVDYHLYP in REDV and DDDEEKFLRRIGRFG in both HSS1 and HSS3, may disrupt the formation of the $\beta$-spirals as they contain no P-G dipeptides.
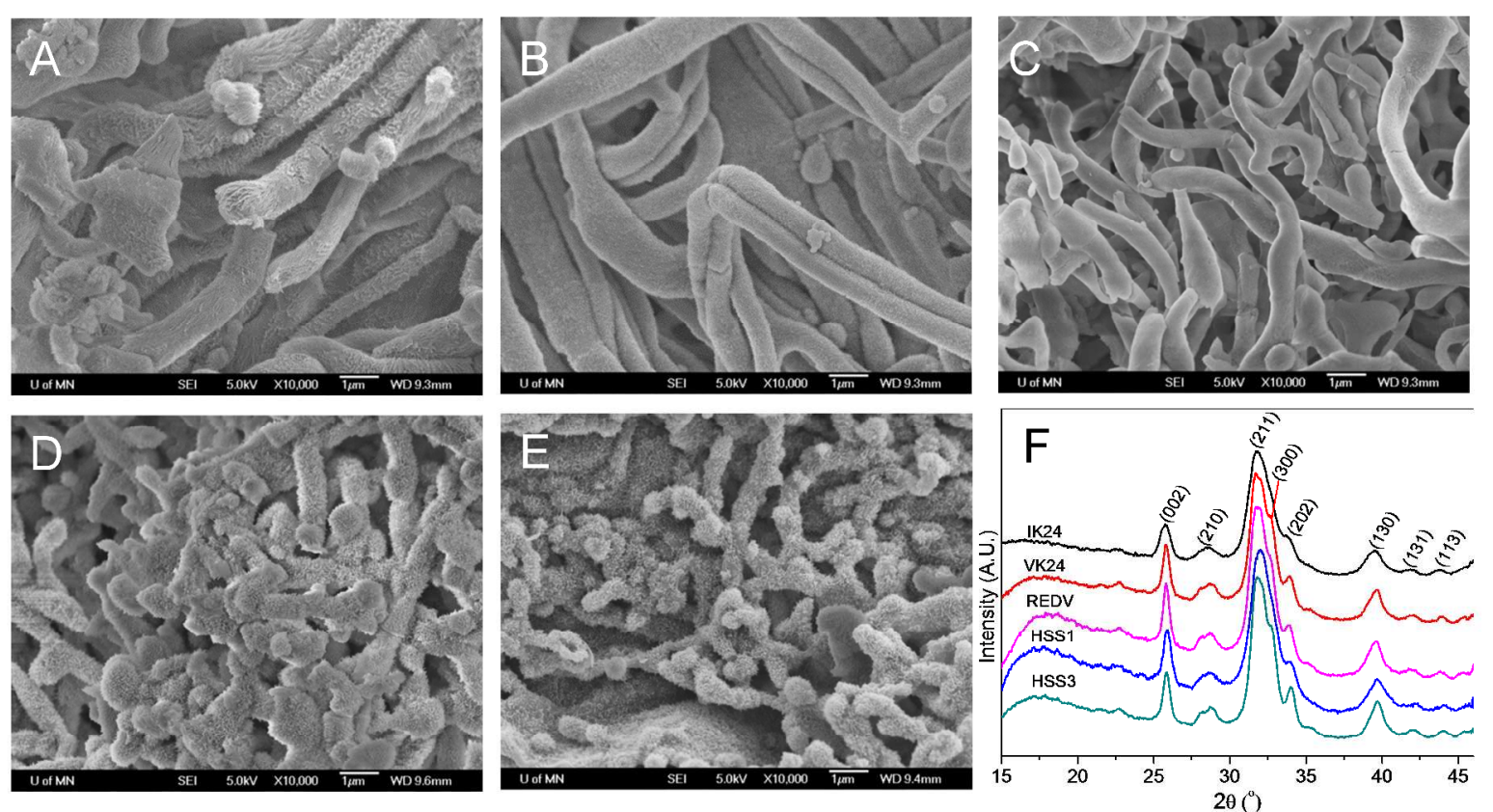

Figure 3. SEM images of the mineralized IK24 (A), VK24 (B), REDV (C), HSS1 (D) and HSS3 (E).

(F) XRD of ELR fibrils after 7 days of mineralization via the PILP process. Peak labels correspond to the crystal planes of HAp.

Intrafibrillar mineralization in ELR fibrils might be driven by capillarity rather than electrostatic attractions between the ELRs and liquid ACP precursor. In free solution, the soluble PASPs bind to calcium and phosphate ions forming nanoclusters. Once the liquid ACP precursor infiltrates into nanocompartments of the ELR fibrils, mineralization rates in the fibrils will be enhanced over those in 
free solution because of the large contact between the nanoclusters and the compartment wall which can reduce the energy barrier for solidification and crystallization of these metastable nanoclusters. ${ }^{47}$

Electrostatic interactions may not be critical for intrafibrillar mineralization. ${ }^{32,48}$ The cationic polyelectrolyte, polyallyamine hydrochloride $(\mathrm{PAH})$, can also stabilize $\mathrm{CaP}$ in its supersaturated solution, forming positively charged nanoclusters. ${ }^{49}$ When positively charged IK24 fibrils were incubated in the PAH stabilized mineralization solution for 3 days, granulation was found on both the periphery and fracture surface of the fibrils (Figure 4C) in contrast to the unmineralized ones (Figure 4A). Collagen fibrils have smooth surface and periodic banding patterns (Figure 4B). They were also successfully mineralized by the positively charged PAH stabilized ACP precursors showing minerals on the fibrils (Figure 4D).
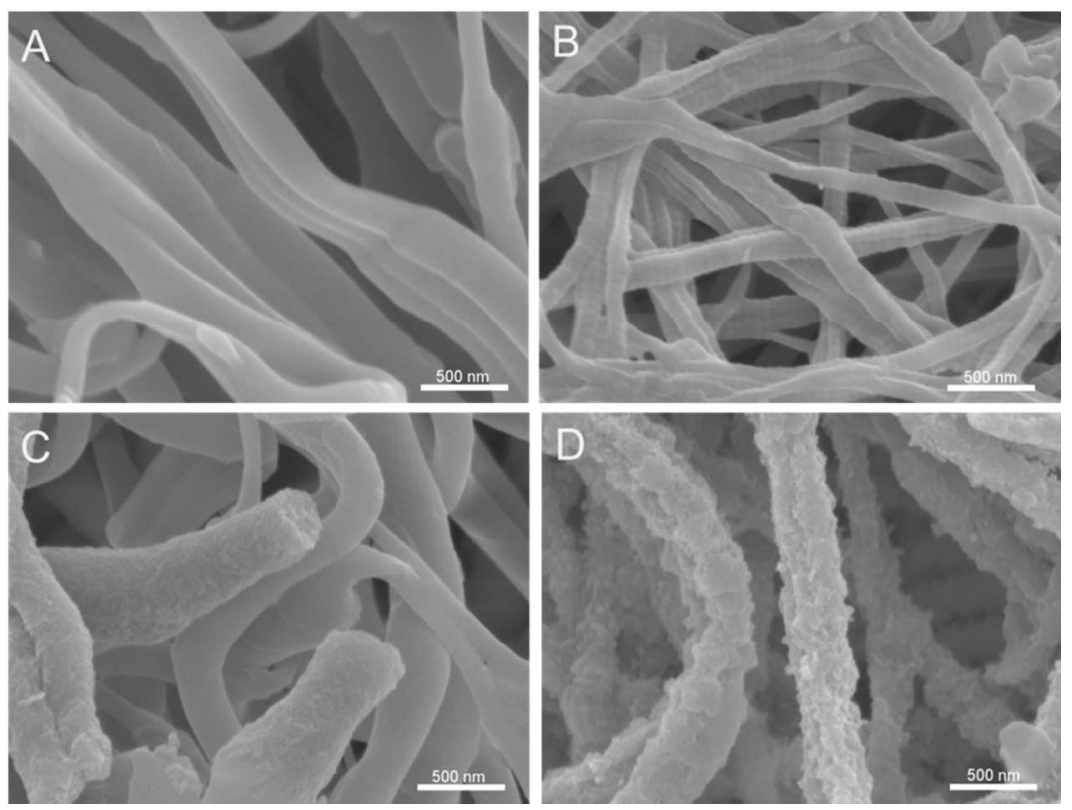

Figure 4. SEM images of IK24 fibrils (A) and collagen fibrils (B), as well as mineralized IK24 (C) and collagen fibrils (D) after 3 days of incubation in the PAH stabilized PILP solution.

In our study, the mineralization solution was refreshed every 3 days. No precipitation was found in the solution but the ELR sponges were mineralized. This result indicates the PASP prevented crystallization of ACP nanoclusters in solution but promoted it in the organic matrix. Each collagen triple helix contains 
$8 \%$ of basic and $11 \%$ of acidic amino acids. ${ }^{50}$ When collagen molecules are laterally registered to form native fibrils with D periodicity, highly positively charged regions are created in gap zones through which the negatively charged ACP nanoclusters are preferentially infiltrated in. ${ }^{18}$ Because all ELRs contained positively charged lysine residues and they were highly positively charged at neutral $\mathrm{pH}$ except REDV, the preferential deposition of ACP nanoclusters into ELR fibrils indicates the electrostatic attraction promote mineral deposition. The isoelectric point of REDV is 5.4 due to the incorporation of the negatively charged sequence-EEIQIGHIPREDVDYHLYP. The smooth surface of the mineralized REDV fibrils suggesting that the negatively charged sequence in REDV suppressed the infiltration of the negatively charged ACP clusters. Compared to the relatively apolar nature of the rest of the recombinamer, the high polarity of the $\mathrm{SN}_{\mathrm{A}} 15$ epitope in HSS1 and HSS3 leads to a preferential segregation of this epitope to the water-matrix interface. This effect has been previously reported on micellar arrangements of amphiphilic ELRs bearing the $\mathrm{SN}_{\mathrm{A}} 15 .{ }^{51}$ This would cause repulsion against the negatively charged ACP nanoclusters. Therefore, overgrowth of the mineral on HSS1 and HSS3 fibrils is likely due to the pre-coalescence of fibrils.

\subsection{Crystal spatial organization in ELR fibrils}

As shown in Figure 5, the HAp nanocrystals in IK24 fibrils were needle-like in shape, less than $100 \mathrm{~nm}$ long and 10-20 $\mathrm{nm}$ wide. They were aligned along the long axis of the fibril. The arc-shaped diffraction of the (002) planes from the corresponding selected area electron diffraction (SAED) pattern indicated that crystals were oriented with their (002) planes parallel along the long axis of the fibril (Figure 5A, S3A and S3B). Additionally, needle-like HAp nanocrystals oriented with their (002) planes roughly perpendicular to the long axis of fibrils and randomly oriented HAp crystals were also observed in some fibrils (Figure 5E, S3C and S3D). In mineralized collagen fibrils, the orientation of HAp crystals has been generally considered to be directed by the stereochemical features of collagen, ${ }^{52}$ whereas the confinement provided by the nanochannels within the fibrils also plays a critical role. ${ }^{5}$ As the collagen triple-helices are aligned parallel in the axial direction of the fibril, crystals growth along the long-axis of 
the fibrils is energetically favorable. ${ }^{17}$ It has been demonstrated that synthetic confinements of track etched alumina membranes with pores of $25 \mathrm{~nm}$ in diameter directed the preferential orientation of HA nanocrystals transformed from ACP. ${ }^{5}$ Oriented mineral growth has been achieved in self-assembled PCL-b-poly(acrylic acid) block copolymers decorated on PCL nanofibers. ${ }^{53}$ Pluronic F127 triblock copolymers that created ordered structures containing periodic and nanoscale aqueous domains also successfully guided the orientation of HAp nanocrystals. ${ }^{19}$ Oriented ultrathin $\mathrm{CaCO}_{3}$ monolayers have also been achieved within the layered nano-gaps of smectic liquid crystalline networks. ${ }^{54}$ In this study, preferentially-oriented HA nanocrystals with their $\left[\begin{array}{lll}0 & 0 & 1\end{array}\right]$ direction aligned roughly parallel along the long axis of ELR fibril occurred as the PASP induced $\mathrm{CaP}$ nanoclusters permeate into the fibrils and grow along the longitudinal direction of the fibrils. Such structure closely resembles that of the mineralized collagen fibrils, i.e., the nanostructure of bone.

Because ELR fibrils have no periodic gap zones as those of collagen fibrils, most of ACP nanoclusters infiltrate into the fibrils from their periphery. Although the nanocrystals tend to be oriented in parallel along the fibrils to minimize capillary forces, HAp nanocrystals can orient randomly or with their [001] perpendicular to the long axis of the fibrils when the rate of crystal growth is faster than the permeation and reorganization of the ACP nanoclusters inside the fibril. In contrast, the ACP nanoclusters that infiltrate through the periodic gap zones of collagen fibrils can grow along the long axis of the fibrils and get aligned. Indeed, when collagen fibrils were poorly organized, the mineral infiltration and crystal orientation were highly interrupted. ${ }^{6}$

In an organic template with a myriad of nanoscale compartments, each nano-compartment can serve as an independent reservoir for mineral deposition. Without the organic matrix, the PASP stabilized ACP nanoclusters are unstable. ${ }^{55}$ These metastable nanoclusters tend to aggregate, coalesce, crystallize and grow into randomly oriented HAp nanocrystals via colloidal aggregation and phase transition. In the presence of the IK24 matrix without self-assembled fibrillar structure, the mineralization was highly 

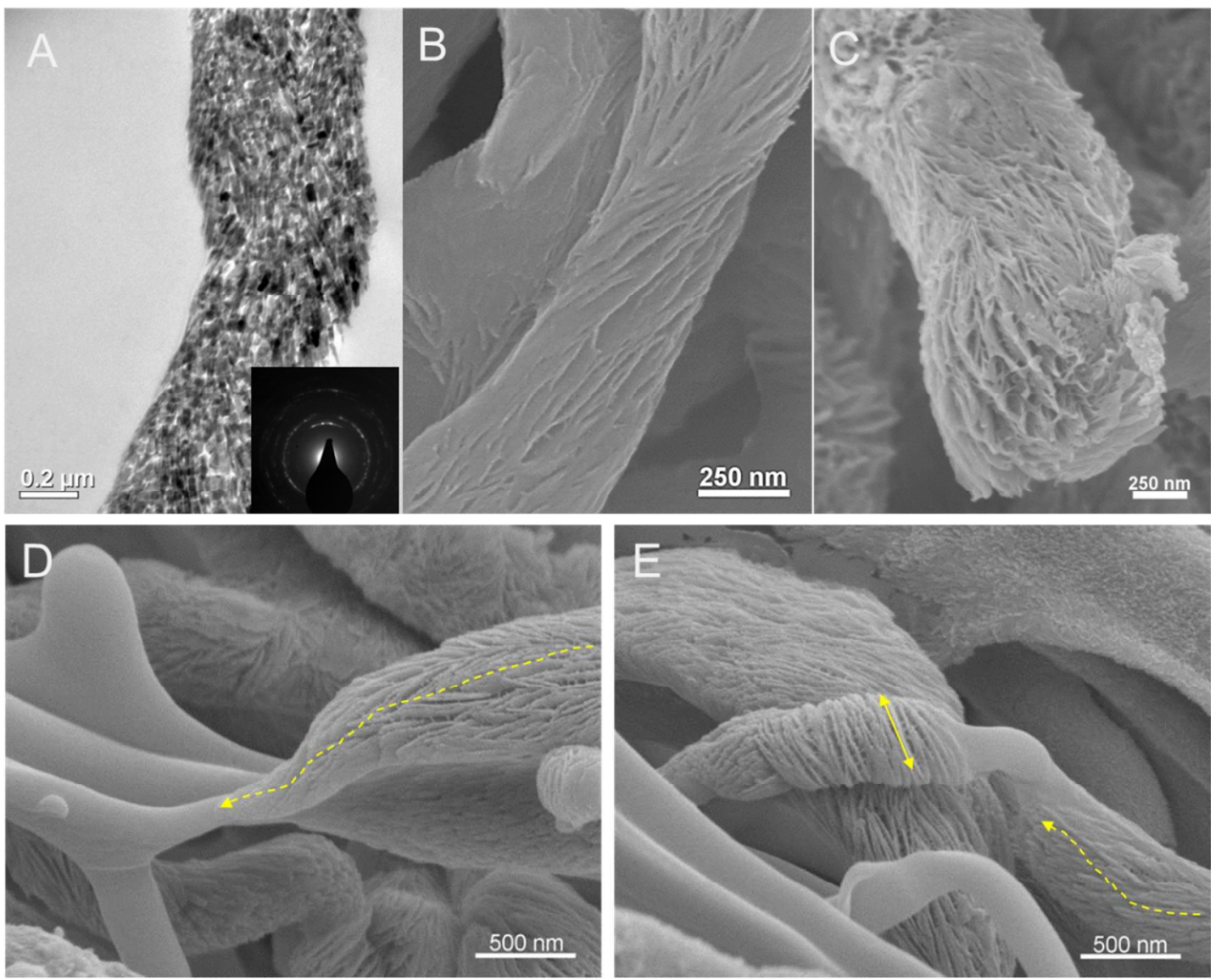

Figure 5. TEM and SEM images of the IK24 fibrils after 7 days of biomimetic mineralization via the PILP process. (A) Bright-field TEM images of the mineralized IK24 fibrils. Inset is a SAED pattern of the corresponding fibrils. The minerals were hydroxyapatite nanocrystals oriented roughly parallel to the longitudinal axis of the fibrils. (B-E) SEM images of mineralized IK24 fibrils. (D-E) SEM images of partially mineralized fibrils with distinct morphological differences between unmineralized and mineralized regions. Solid line with two arrows indicates the crystal orientation and dash line with an arrow indicate the mineral growing direction. 


\section{Conclusions}

In summary, we have successfully achieved the intrafibrillar mineralization of ELR fibrils using a bioinspired approach learned from collagen mineralization, by which a stable inorganic-organic nanocomposite that mimics the nanostructure of bone, i.e. intrafibrillarly mineralized collagen fibrils, was obtained. Through thermally driven self-assembly, the ELR molecules spontaneously aggregated into fibrils composed of bundles of filaments. Polymer induced calcium phosphate precursors infiltrated into the interstitial spaces of the ELR fibrils and formed needle-like HA nanocrystals. In particular, these recombinamers composed of only (VPGXG)n domains (IK24 andVK24) showed thoroughly and homogeneously intrafibrillar mineralization, whereas other recombinamers (REDV, HSS1 and HSS3) that contained additional bioactive sequences disturbed their initial $\beta$-spiral self-assembly and demonstrated different mineralization feature. These results indicate that the self-assembled superstructure, i.e., a continuum $\beta$-spiral structure and an unperturbed fibrillar structure, rather than electrostatic interactions or bioactive sequences in the recombinamers, play the key role in the intrafibrillar mineralization. Accomplishment of the intrafibrillar mineralization in ELRs fibrils provides a new approach to the development of bone-like biomaterials. It also brings new insights into other biomineralization systems, i.e., the elastin-related paths that lead to pathogenic vascular calcification. ${ }^{56-57}$ As the recombinant technology enables designing and producing well-defined ELRs, the superstructure and surface charges can be fine-tuned to explore their role in mineralization.

Conflict of Interest: The authors declare no competing financial interest

Supporting Information Available: Detailed experimental procedures and SEM, FTIR and TEM data. This material is available free of charge via the Internet at http://pubs.acs.org.

Acknowledgement. This project was partially supported by a 3M Foundation NonTenured Faculty Award to C.A.. J.C.R.-C. wishes to thank the European Commission (HEALTH-F4-2011-278557, PITN-GA- 
2012-317306, MSCA-ITN-2014-642687 and NMP-2014-646075), The Ministry of Economy and Competitiveness (Spain) (MAT2013-42473-R and MAT2015-68901R), and the Junta de Castilla y Leon (VA244U13, VA313U14 and VA015U16). Parts of this work were carried out in the University of Minnesota I.T. Characterization Facility, which receives partial support from NSF through the MRSEC program.

\section{REFERENCES}

1. Weiner, S.; Wagner, H. D. The Material Bone: Structure Mechanical Function Relations. Annu. Rev. Mater. Sci. 1998, 28, 271-298.

2. Fratzl, P.; Gupta, H. S.; Paschalis, E. P.; Roschger, P. Structure and Mechanical Quality of the Collagen-mineral Nano-composite in Bone. J. Mater. Chem. 2004, 14, 2115-2123.

3. Landis, W. J.; Hodgens, K. J.; Arena, J.; Song, M. J.; McEwen, B. F. Structural Relations between Collagen and Mineral in None as Determined by High Voltage Electron Microscopic Tomography. Microsc. Res. Tech. 1996, 33, 192-202.

4. Olszta, M. J.; Cheng, X. G.; Jee, S. S.; Kumar, R.; Kim, Y. Y.; Kaufman, M. J.; Douglas, E. P.; Gower, L. B. Bone Structure and Formation: A New Perspective. Mater. Sci. Eng. R 2007, 58, 77-116. 5. Cantaert, B.; Beniash, E.; Meldrum, F. C. Nanoscale Confinement Controls the Crystallization of Calcium Phosphate: Relevance to Bone Formation. Chem. - Eur. J. 2013, 19, 14918-14924.

6. Nudelman, F.; Bomans, P. H. H.; George, A.; de With, G.; Sommerdijk, N. A. J. M. The Role of the Amorphous Phase on the Biomimetic Mineralization of collagen. Faraday Discuss. 2012, 159, 357370.

7. Mahamid, J.; Sharir, A.; Addadi, L.; Weiner, S. Amorphous Calcium Phosphate Is a Major Component of the Forming Fin Bones of Zebrafish: Indications for an Amorphous Precursor Phase. $P$. Natl. Acad. Sci. U. S. A. 2008, 105, 12748-12753. 
8. Weiner, S.; Sagi, I.; Addadi, L. Choosing the Crystallization Path less Traveled. Science 2005, $309,1027-1028$.

9. Shoulders, M. D.; Raines, R. T. Collagen Structure and Stability. Annu. Rev. Biochem. 2009, 78, 929-958.

10. Hulmes, D. J. S.; Miller, A. Quasi-hexagonal Molecular Packing in Collagen Fibrils. Nature 1979, $282,878-880$.

11. Trus, B. L.; Piez, K. A. Compressed Microfibril Models of the Native Collagen Fibril. Nature 1980, 286, 300-301.

12. Orgel, J. P. R. O.; Irving, T. C.; Miller, A.; Wess, T. J. Microfibrillar Structure of Type I Collagen In Situ. P. Natl. Acad. Sci. U. S. A. 2006, 103, 9001-9005.

13. Hodge, A. J.; Petruska, J. A., in Aspects of Protein Strucutre. Academic: New York, 1963.

14. George, A.; Veis, A. Phosphorylated Proteins and Control over Apatite Nucleation, Crystal Growth, and Inhibition. Chem. Rev. 2008, 108, 4670-4693.

15. Jee, S. S.; Kasinath, R. K.; DiMasi, E.; Kim, Y. Y.; Gower, L. Oriented Hydroxyapatite in Turkey Tendon Mineralized via the Polymer-Induced Liquid-Precursor (PILP) Process. Crystengcomm 2011, 13, $2077-2083$.

16. Deshpande, A. S.; Beniash, E. Bioinspired Synthesis of Mineralized Collagen Fibrils. Cryst. Growth Des. 2008, 8, 3084-3090.

17. Nudelman, F.; Lausch, A. J.; Sommerdijk, N. A. J. M.; Sone, E. D. In Vitro Models of Collagen Biomineralization. J. Struct. Biol. 2013, 183, 258-269.

18. Nudelman, F.; Pieterse, K.; George, A.; Bomans, P. H. H.; Friedrich, H.; Brylka, L. J.; Hilbers, P. A. J.; de With, G.; Sommerdijk, N. A. J. M. The Role of Collagen in Bone Apatite Formation in the Presence of Hydroxyapatite Nucleation Inhibitors. Nat. Mater. 2010, 9, 1004-1009.

19. Chen, J. L.; Chu, B.; Hsiao, B. S. Mineralization of Hydroxyapatite in Electrospun Nanofibrous Poly(L-Lactic Acid) Scaffolds. J. Biomed. Mater. Res., Part A 2006, 79A, 307-317. 
20. Liao, S.; Murugan, R.; Chan, C. K.; Ramakrishna, S. Processing Nanoengineered Scaffolds through Electrospinning and Mineralization, Suitable for Biomimetic Bone Tissue Engineering. J. Mech. Behav. Biomed. Mater. 2008, 1, 252-260.

21. Yu, H. S.; Hong, S. J.; Kim, H. W. Surface-mineralized Polymeric Nanofiber for the Population and Osteogenic Stimulation of Rat Bone-marrow Stromal Cells. Mater. Chem. Phys. 2009, 113, 873-877. 22. Veis, A. Mineralization in Organic Matrix Frameworks. Rev. Mineral. Geochem. 2003, 54, 249289.

23. Urry, D. W. Molecular machines - How Motion and other Functions of Living Organisms Can Result from Reversible Chemical-changes. Angew. Chem., Int. Ed. Engl. 1993, 32, 819-841.

24. Urry, D. W.; Okamoto, K.; Harris, R. D.; Hendrix, C. F.; Long, M. M. Synthetic, Cross-linked Polypentapeptide of Tropoelastin - Anisotropic, Fibrillar Elastomer. Biochemistry 1976, 15, 4083-4089.

25. Bellingham, C. M.; Lillie, M. A.; Gosline, J. M.; Wright, G. M.; Starcher, B. C.; Bailey, A. J.; Woodhouse, K. A.; Keeley, F. W. Recombinant Human Elastin Polypeptides Self-assemble into Biomaterials with Elastin-like Properties. Biopolymers 2003, 70, 445-455.

26. Bressan, G. M.; Pasqualironchetti, I.; Fornieri, C.; Mattioli, F.; Castellani, I.; Volpin, D. Relevance of Aggregation Properties of Tropoelastin to the Assembly and Structure of Elastic fibers. $J$. Ultrastruct. Mol. Struct. Res. 1986, 94, 209-216.

27. Urry, D. W. Physical Chemistry of Biological Free Energy Transduction as Demonstrated by Elastic Protein-based Polymers. J. Phys. Chem. B 1997, 101, 11007-11028.

28. Massia, S. P.; Hubbell, J. A. Vascular Endothelial-cell Adhesion and Spreading Promoted by the Reptide REDV of the IIICS Region of Plasma Fibronectin Is Mediated by integrin Alpha-4-Beta 1. J. Biol. Chem. 1992, 267, 14019-14026.

29. Urry, D. W.; Parker, T. M. Mechanics of Elastin: Molecular Mechanism of Biological Elasticity and its Relationship to Contraction. J. Muscle Res. Cell Motil. 2002, 23, 543-559. 
30. Raj, P. A.; Johnsson, M.; Levine, M. J.; Nancollas, G. H. Salivary Statherin - Dependence on Sequence, Charge, Hydrogen-bonding Potency, and Helical Conformation for Adsorption to Hydroxyapatite and Inhibition of Mineralization. J. Biol. Chem. 1992, 267, 5968-5976.

31. Misbah, M. H.; Espanol, M.; Quintanilla, L.; Ginebra, M. P.; Rodriguez-Cabello, J. C. Formation of Calcium Phosphate Nanostructures under the Influence of Self-assembling Hybrid Elastin-likestatherin Recombinamers. RSC Adv. 2016, 6, 31225-31234.

32. Li, Y. P.; Chen, X.; Fok, A.; Rodriguez-Cabello, J. C.; Aparicio, C. Biomimetic Mineralization of Recombinamer-based Hydrogels toward Controlled Morphologies and High Mineral Density. ACS Appl. Mater. Interfaces 2015, 7, 25784-25792.

33. Savitzky, A.; Golay, M. J. E. Smoothing and Differentiation of Data by Simplified Least Squares Procedures. Anal. Chem. 1964, 36, 1627-1639.

34. Bochicchio, B.; Pepe, A. Role of Polyproline II Conformation in Human Tropoelastin Structure. Chirality 2011, 23, 694-702.

35. Mouro, C.; Bondon, A.; Simonneaux, G.; Jung, C. H-1-NMR Study of Diamagnetic Cytochrome P450cam: Assignment of Heme Resonances and Substrate Dependance of One Cysteinate Beta Proton. FEBS Lett. 1997, 414, 203-208.

36. Jackson, M.; Mantsch, H. H. The Use and Misuse of FTIR Spectroscopy in the Determination of Protein-structure. Crit. Rev. Biochem. Mol. Biol. 1995, 30, 95-120.

37. Goormaghtigh, E.; Cabiaux, V.; Ruysschaert, J. M. Secondary Structure and Dosage of Soluble and Membrane-proteins by Attenuated Total Reflection Fourier-transform Infrared-spectroscopy on Hydrated Films. Eur. J. Biochem. 1990, 193, 409-420.

38. Serrano, V.; Liu, W.; Franzen, S. An Infrared Spectroscopic Study of the Conformational Transition of Elastin-like Polypeptides. Biophys. J. 2007, 93, 2429-2435.

39. Nicolini, C.; Ravindra, R.; Ludolph, B.; Winter, R. Characterization of the Temperature- and Pressure-induced Inverse and Reentrant Transition of the Minimum Elastin-like Polypeptide GVG(VPGVG) by DSC, PPC, CD, and FT-IR Spectroscopy. Biophys. J. 2004, 86, 1385-1392. 
40. Urry, D. W. Entropic Elastic Processes in Protein Mechanisms .2. Simple (Passive) and Coupled (Active) Development of Elastic Forces. J. Protein Chem. 1988, 7, 81-114.

41. Urry, D. W. Entropic Elastic Processes in Protein Mechanisms. 1. Elastic Structure due to an Inverse Temperature Transition and Elasticity due to Internal Chain Dynamics. J. Protein Chem. 1988, 7 , $1-34$.

42. Volpin, D.; Urry, D. W.; Pasqualironchetti, I.; Gotte, L. Studies by Electron-microscopy on Structure of Coacervates of Synthetic Polypeptides of Tropoelastin. Micron 1976, 7, 193-198.

43. Volpin, D.; Urry, D. W.; Cox, B. A.; Gotte, L. Optical Diffraction of Tropoelastin and AlphaElastin Coacervates. Biochim. Biophys. Acta 1976, 439, 253-258.

44. Misbah, M. H.; Quintanilla, L.; Alonso, M.; Rodrguez-Cabello, J. C. Evolution of Amphiphilic Elastin-like Co-recombinamer Morphologies from Micelles to a Lyotropic Hydrogel. Polymer 2015, 81, $37-44$.

45. Bleek, K.; Taubert, A. New Developments in Polymer-controlled, Bioinspired Calcium Phosphate Mineralization from Aqueous Solution. Acta Biomater. 2013, 9, 6283-6321.

46. Li, Y. P.; Chen, X.; Ribeiro, A. J.; Jensen, E. D.; Holmberg, K. V.; Rodriguez-Cabello, J. C.; Aparicio, C. Hybrid Nnotopographical Surfaces Obtained by Biomimetic Mineralization of Statherininspired Elastin-like Recombinamers. Adv. Healthcare Mater. 2014, 3, 1638-1647.

47. Cantaert, B.; Beniash, E.; Meldrum, F. C. The Role of Poly(Aspartic Acid) in the Precipitation of Calcium Phosphate in Confinement. J. Mater. Chem. B 2013, 1, 6586-6595.

48. Niu, L. N.; Jee, S. E.; Jiao, K.; Tonggu, L.; Li, M.; Wang, L.; Yang, Y. D.; Bian, J. H.; Breschi, L.; Jang, S. S.; Chen, J. H.; Pashley, D. H.; Tay, F. R. Collagen Intrafibrillar Mineralization as a Result of the Balance between Osmotic Equilibrium and Electroneutrality. Nat. Mater. 2016.

49. Jiao, K.; Niu, L. N.; Ma, C. F.; Huang, X. Q.; Pei, D. D.; Luo, T.; Huang, Q.; Chen, J. H.; Tay, F. R. Complementarity and Uncertainty in Intrafibrillar Mineralization of collagen. Adv. Funct. Mater. 2016, $26,6858-6875$.

50. Piez, K. A.; Reddi, A. H., Extracellular Matrix Biochemistry. 1984. 
51. Prieto, S.; Shkilnyy, A.; Rumplasch, C.; Ribeiro, A.; Arias, F. J.; Rodriguez-Cabello, J. C.; Taubert, A. Biomimetic Calcium Phosphate Mineralization with Multifunctional Elastin-like Recombinamers. Biomacromolecules 2011, 12, 1480-1486.

52. Silver, F. H.; Landis, W. J. Deposition of Apatite in Mineralizing Vertebrate Extracellular Matrices: A Model of Possible nucleation Sites on Type I Collagen. Connect. Tissue Res. 2011, 52, 242254.

53. He, W. X.; Rajasekharan, A. K.; Tehrani-Bagha, A. R.; Andersson, M. Mesoscopically Ordered Bone-mimetic Nanocomposites. Adv. Mater. 2015, 27, 2260-2264.

54. Xu, Y. F.; van Kuringen, H. P. C.; Mulder, D. J.; Schenning, A. P. H. J.; Sommerdijk, N. A. J. M. Smectic Liquid Crystal Polymers as a Template for Ultrathin CaCO3 Nanolayers. RSC Adv. 2016, 6, 13953-13956.

55. Li, Y. P.; Aparicio, C. Discerning the Subfibrillar Structure of Mineralized Collagen Fibrils: A Model for the Ultrastructure of Bone. Plos One 2013, 8 .

56. Bertazzo, S.; Gentleman, E.; Cloyd, K. L.; Chester, A. H.; Yacoub, M. H.; Stevens, M. M. Nanoanalytical Electron Microscopy Reveals Fundamental Insights into Human Cardiovascular Tissue Calcification. Nat. Mater. 2013, 12, 576-583.

57. Reznikov, N.; Steele, J. A. M.; Fratzl, P.; Stevens, M. M. A Materials Science Vision of Extracellular Matrix Mineralization. Nat. Rev. Mater. 2016, 1, 1-14. 
